\title{
Optimal scheduling strategy for virtual power plants based on credibility theory
}

\author{
Qian Ai, Songli Fan and Longjian Piao
}

\begin{abstract}
The virtual power plant (VPP) is a new and efficient solution to manage the integration of distributed energy resources (DERs) into the power system. Considering the unpredictable output of stochastic DERs, conventional scheduling strategies always set plenty of reserve aside in order to guarantee the reliability of operation, which is too conservative to gain more benefits. Thus, it is significant to research the scheduling strategies of VPPS, which can coordinate the risks and benefits of VPP operation. This paper presents a fuzzy chance-constrained scheduling model which utilizes fuzzy variables to describe uncertain features of distributed generators (DGs). Based on credibility theory, the concept of the confidence level is introduced to quantify the feasibility of the conditions, which reflects the risk tolerance of VPP operation. By transforming the fuzzy chance constraints into their equivalent forms, traditional optimization algorithms can be used to solve the optimal scheduling problem. An IEEE 6-node system is employed to prove the feasibility of the proposed scheduling model. Case studies demonstrate that the fuzzy chance strategy is superior to conservative scheduling strategies in realizing the right balance between risks and benefits.
\end{abstract}

Keywords: Credibility theory, Distributed energy resource (DER), Scheduling strategy, Uncertain factors, Virtual power plant (VPP)

\section{Introduction}

Nowadays, due to the rising prices of fossil fuels and the threat of climate change caused by greenhouse gases, distributed energy resources (DERs) have drawn widespread attention because of their clean and renewable characteristics [1-3]. However, the output of DERs is fluctuating and unpredictable. As the penetration of intermittent renewables in the grids is increasing gradually, more technical challenges need to be addressed in the schedule and control of their operation [4-7]. Meanwhile, the liberalization of the electricity market makes DERs inevitable. However, the small capacity, intermittent output and the lack of appropriate interaction with the system operator are the biggest barriers ahead of DERs for participation in the electricity market. To solve these problems mentioned above, the DERs could be aggregated as an entity which can behave like a conventional generator, naming the virtual power plant (VPP) [8-10].

\footnotetext{
* Correspondence: aiqian@sjtu.edu.cn

Department of Electrical Engineering, Shanghai Jiao Tong University, Shanghai 200240, China
}

In $[11,12]$, a VPP is defined as a coalition of DERs including distributed generations (DGs), storage devices, and interruptible loads. Considering the characteristics of each DER and the impact of network, VPPs generate one unit portfolio which can be utilized to offer services to the system operator, and even to make contracts in the wholesale market. Therefore, by introducing the concept of the VPP, the visibility and controllability of DERs for system operators will be improved considerably, just the same as the conventional transmissionconnected power plants.

To realize the concept of the VPP, scholars around the world have done lots of studies on VPPs in many aspects [13-17], including VPP modeling methods, negotiating behaviors in the market, bidding strategies, reliability evaluation, management systems, and so on. Among them, the optimal scheduling strategy of DERs in the VPP is a hot topic. Reference [18] proposed a nonequilibrium model which takes into account the supply and demand balancing constraint and security constraint of VPPs. On this basis, the strategy proposed in [19] considered the effect of reliability and determined the optimum hourly operating strategy of DERs by applying 
the Monte Carlo simulation method. However, these scheduling strategies are based on the deterministic market prices. Considering the uncertainties in prices, [20] developed a new risk constrained scheduling for VPPs to help the aggregator bid in the day-ahead market.

However, the optimal bidding strategy of those researches mainly focused on maximizing the VPP's profit in different types of markets. With regard to reserve level, these methods spare large capacity as recovery to balance the supply and demand. Although the system stability can be guaranteed in this deterministic way, the obtained scheduling results are always inevitably conservative. Actually, more benefits can be gained via reducing the reserve capacity appropriately.

This paper proposes a fuzzy chance-constrained scheduling model to provide solutions for the optimal scheduling strategy for VPPs based on credibility theory. In this model, as the prediction errors of renewables are characterized as fuzzy parameters, the related constraints correspondingly contain fuzzy variables which need to be tackled properly. By introducing the concept of the confidence level, the feasibility of the conditions where the fuzzy chance constraints can be satisfied is quantified, so as to characterize the risk tolerance of VPPs. The proposed fuzzy chance model is difficult to solve due to the incorporation of fuzzy variables. By transforming the constraints containing fuzzy variables into their equivalent forms, the chance constraints are converted into deterministic constraints which consider the fuzzy risks (or reliability) as well. Then traditional optimization algorithms can be utilized to solve the optimal scheduling problem, in order to achieve a compromise between risks and benefits.

The rest of the paper is organized as follows: the theoretical basis of this paper is presented in Credibility theory and fuzzy chance-constrained programming, including credibility theory and fuzzy chance constrained programming; the proposed VPP scheduling strategy based on credibility theory is demonstrated in VPP scheduling strategy based on credibility theory; solution methods used in this paper are stated in Methods; case studies and discussion are given in Case study; and finally, the conclusion is drawn in Conclusions.

\section{Credibility theory and fuzzy chance-constrained programming}

Distinguished from the conventional power plants, the scheduling of VPPs is required to deal with a number of uncertain variables from renewables and loads. The common ways to process those uncertain variables are categorized into determinate and indeterminate methods. Determinate method is to set plenty of reserve aside in order to guarantee the reliability of operation. However, this approach is too conservative to coordinate the risks and benefits of VPP operation. More benefits can be obtained by ignoring the small probability events and making decisions within the scope of risk tolerance [21]. The fuzzy chance-constrained programming method proposed in this paper is one kind of indeterminate scheduling strategy.

In this approach, the decision result is allowed to violate the constraints which contain fuzzy variables, but the feasibility of satisfying the constraints should be no less than the preset confidence level. The confidence level is a concept which reflects the risk tolerance of the system, or the reliability requirements of the system when facing uncertain variables. In conclusion, the optimal fuzzy chance-constrained scheduling strategy is a compromise between risks and economic profits. Credibility theory provides theoretical support to solve the confidence level problem of the fuzzy chance strategy decision, which greatly contributes to the development and improvement of the fuzzy chance programming theory.

\section{Credibility theory}

The concept of the fuzzy set theory was initiated by Zadeh via the membership function [22]. In order to measure a fuzzy event, the possibility measure is proposed. Since then the possibility theory has been studied by many researchers. Although the possibility measure has been widely used, it does not possess the self-duality property. Thus, if the possibility measure equals one it does not mean the event will happen definitely, while the event may happen even though the possibility measure is zero. So the solution to the fuzzy decision problem has been a mathematical conundrum for a long time.

Credibility theory was propounded by Liu in 2004 as a branch of mathematics for studying the fuzzy behavior [23]. It establishes a complete axiomatic system which is parallel with probability theory in dealing with uncertainty. Based on five axioms mentioned in [23], a credibility measure is defined.

According to Liu [23], the following five axioms should hold to ensure that the number $\mathrm{Cr}(\mathrm{A})$ has certain mathematical properties.

\section{Definition 2.1:}

Axiom 1: $\operatorname{Cr}\{\Theta\}=1$;

Axiom 2: $\mathrm{Cr}$ is non-decreasing, i.e., if $\mathrm{A} \subseteq \mathrm{B}$, there is always $\operatorname{Cr}\{\mathrm{A}\} \leq \mathrm{Cr}\{\mathrm{B}\}$;

Axiom 3: Cr is self-dual: $\forall \mathrm{A} \in \mathrm{P}(\Theta), \mathrm{Cr}\{\mathrm{A}\}+\operatorname{Cr}\left\{\mathrm{A}^{\mathrm{C}}\right\}=1$; Axiom 4: $\forall \mathrm{A}_{i} \in\left\{\mathrm{A}_{i} \mid \operatorname{Cr}\left\{\mathrm{A}_{i}\right\} \leq 0.5\right\}$, there is $\operatorname{Cr}\left\{\mathrm{U}_{i} \mathrm{~A}_{i}\right\} \wedge$ $0.5=\sup _{i} \operatorname{Cr}\left\{\mathrm{A}_{i}\right\}$.

Axiom 5: Let $\Theta_{1}, \Theta_{2}, \ldots, \Theta_{n}$ be the nonempty sets corresponding to that $\mathrm{Cr}_{1}, \mathrm{Cr}_{2}, \ldots, \mathrm{Cr}_{n}$ satisfy the axioms as respectively defined above, and let $\Theta=\Theta_{1} \times \Theta_{2} \times \ldots \times \Theta_{n}$. 
Then, we have $\operatorname{Cr}\left\{\left(\theta_{1}, \theta_{2}, \ldots, \theta_{n}\right)\right\}=\operatorname{Cr}_{1}\left\{\theta_{1}\right\} \wedge$ $\mathrm{Cr}_{2}\left\{\theta_{2}\right\} \wedge \ldots \wedge \mathrm{Cr}_{n}\left\{\theta_{n}\right\}$ for each $\left(\theta_{1}, \theta_{2}, \ldots, \theta_{n}\right) \in \Theta$. where $\Theta$ is a nonempty set; $\mathrm{P}(\Theta)$ is the possibility set of $\Theta$; the element of $\mathrm{P}(\Theta)$ is a fuzzy event; $\mathrm{A}$ is the subset of $\Theta ; \operatorname{Cr}(\mathrm{A})$ is a non-negative number indicating the credibility of Event A which will occur; and ${ }^{\wedge}$ represents the minimal operator.

Accordingly, the credibility measure describes the credibility level of a fuzzy event; parallel with the confidence level in probability theory, it satisfies the self-duality. It means that the event whose credibility measure is 1 will definitely occur, and similarly, the event whose credibility measure is 0 will never happen. So credibility theory solves the confusion caused by a subordinate degree, and provides theoretical foundations for the fuzzy decision.

In the fuzzy decision of VPP scheduling, some definitions and theorems of credibility theory are utilized, as listed below:

Definition 2.2 (Membership Function): The membership function $\mu(\mathrm{x})$ of the fuzzy parameter $\xi$ is defined as:

$$
\mu(\mathrm{x})=\operatorname{Pos}\{\theta \in \Theta \mid \xi(\theta) \leq \mathrm{x}\}
$$

Definition 2.3 (Credibility Measure): The credibility measure can be obtained from the membership function, which is named as the credibility inversion theorem:

$$
\operatorname{Cr}\{\xi \in \mathrm{A}\}=\frac{\sup \mu(\mathrm{x})_{\mathrm{x} \in \mathrm{A}}+1-\sup \mu(\mathrm{x})_{\mathrm{x} \in \mathrm{A}^{\mathrm{c}}}}{2}
$$

\section{Fuzzy chance-constrained programming}

Fuzzy chance-constrained programming refers to a type of decision-making problem which contains fuzzy parameters. The forward-looking decision results of this problem may not satisfy the constraints with fuzzy parameters to some extent, but the possibility of satisfying the constraints should be no less than the pre-specified confidence level. The general form of the fuzzy chanceconstrained programming problem is:

$$
\left\{\begin{array}{l}
\max \bar{f} \\
\operatorname{Cr}\{f(\boldsymbol{x}, \boldsymbol{\xi}) \geq \bar{f}\} \geq \alpha \\
\operatorname{Cr}\left\{g_{i}(\boldsymbol{x}, \boldsymbol{\xi}) \leq 0, i=1,2, \cdots, n\right\} \geq \beta
\end{array}\right.
$$

where $x$ is the decision vector; $\xi$ is the fuzzy parameter vector; $g_{i}(\boldsymbol{x}, \boldsymbol{\xi}) \leq 0$ is the $i$ th constraint; $\alpha$ is the confidence level of the object function; $\beta$ is the confidence level of the fuzzy constraint. The decision vector $\boldsymbol{x}$ is feasible only when the credibility of $g_{i}(\boldsymbol{x}, \boldsymbol{\xi}) \leq 0$ is no less than $\beta$. Additionally, the optimal solution can maximize the object function $f$ at the confidence level of $\alpha$.

\section{VPP scheduling strategy based on credibility theory \\ Traditional VPP scheduling strategy}

The primary objectives of the VPP optimal scheduling problem are various, such as minimizing the cost of producing energy, or maximizing the profits of VPPs. In this paper, the priority of VPP operation is to make use of the renewable energy resources (RES), and based on this, the objective function is to minimize the costs of conventional unit generation and the interruptible load.

$$
\begin{aligned}
& \min f=\sum_{t=1}^{T}\left\{\sum_{i=1}^{N_{G}}\left[J_{G i}(t)+u_{i}(t-1) u_{i}(t) S U_{i}(t)\right]+\sum_{j=1}^{N_{D R}} J_{D R j}(t)\right\} \\
& J_{G i}(t)=u_{i}(t)\left(a_{i} P_{G i}{ }^{2}+b_{i} P_{G i}+c_{i}\right) \\
& J_{D R j}(t)=d_{j} P_{D R j} u_{j}(t)
\end{aligned}
$$

where $J_{G i}(t)$ is the operational cost of unit $i$ at time $t$; $S U_{i}(t)$ is the start-up cost of the unit $i$ at time $t ; a_{i}, b_{i}, c_{i}$ are the coefficients of the operational cost of the unit $i ; d_{j}$ is the coefficient of the cost by curtailing the load $j ; P_{G i}$ is the output power of the unit $i ; P_{D R j}$ is the virtual generation via curtailing the load; $u_{i}(t)$ is the binary state variable of the unit $i$ at time $t$ : it equals " 1 " if the unit $i$ is on at time $t$, and equals " 0 " if the unit $i$ is off at time $t$. Similarly, $u_{j}(t)$ is the binary state variable of the load $j$ at time $t . N_{G}$ and $N_{D R}$ are the numbers of conventional units and loads in the demand response respectively.

The constraints considered in this model are presented as follows:

1) Supply-demand balancing constraint

$$
\sum_{i=1}^{N_{G}} P_{G i}(t)+\sum_{j=1}^{N_{D R}} P_{D R j}(t)+P_{R E S}(t)=P_{L}(t)
$$

2) System reserve constraint

$$
\sum_{i=1}^{N_{G}} P_{G i}^{\max }+\sum_{j=1}^{N_{D R}} P_{D R j}^{\max }+P_{R E S}(t) \geq P_{L}(t)+R(t)
$$

3) DG constraints

$$
\begin{aligned}
& u_{i}(t) P_{G i}^{\min } \leq P_{G i}(t) \leq u_{i}(t) P_{G i}^{\max } \\
& -R D_{i} \leq P_{G i}(t)-P_{G i}(t-1) \leq R U_{i}
\end{aligned}
$$

4) Interruptible load constraint

$$
u_{j}(t) P_{D R j}^{\min } \leq P_{D R j}(t) \leq u_{j}(t) P_{D R j}^{\max }
$$

where $P_{R E S}(t)$ is the power generated by renewables at time $t ; P_{L}(t)$ is the total load at time $t ; R(t)$ is the reserve 


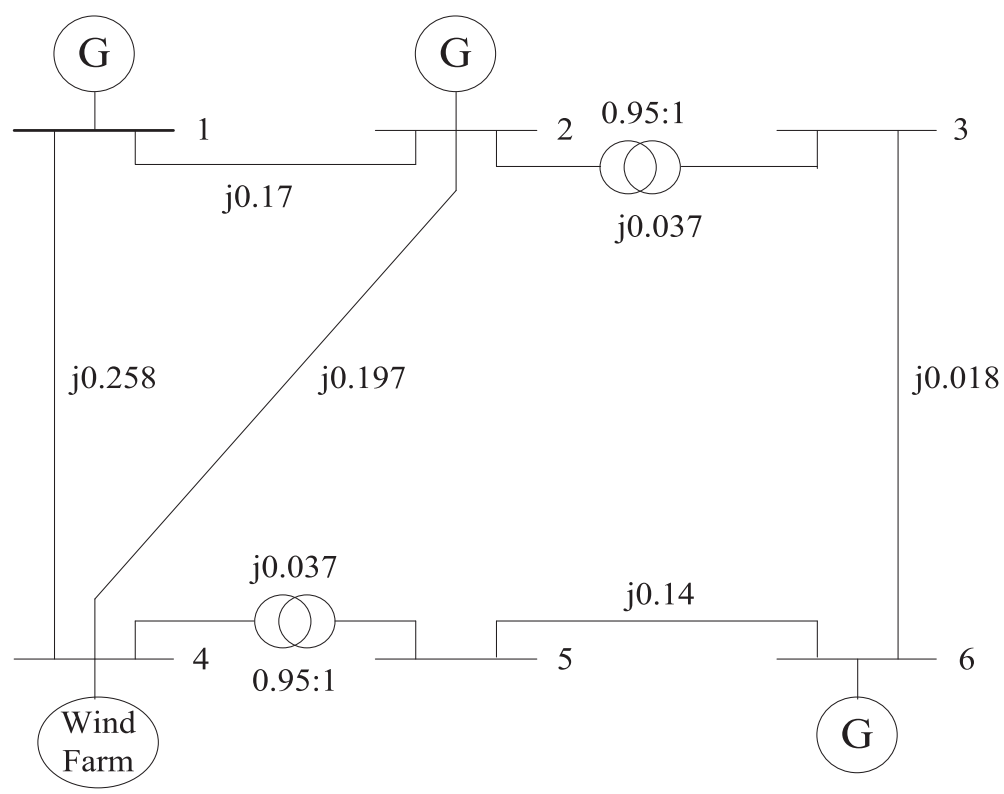

Fig. 1 Wiring Diagram of the VPP

capacity for the fluctuation of load and renewables at time $t ; P_{G i}^{\min }$ and $P_{G i}^{\max }$ are the minimum and the maximum output limit of the unit $i$ respectively; $R U_{i}$ and $R D_{i}$ are the ramp-up rate and the ramp-down rate of the unit $i ; P_{D R j}^{\min }$ and $P_{D R j}^{\max }$ are the minimum and maximum allowed curtailed value of the load $j$ respectively.

\section{Fuzzy chance-constrained VPP scheduling}

The fuzzy parameters of VPP scheduling strategy come from the unpredictable output of renewables in VPPs [24]. Accurate mathematical expressions of those uncertain characteristics should be established, which are the foundations for the uncertain scheduling strategy. The main characterizing methods include modeling output of renewables and modeling their predicted errors. In this paper, the modeling of the forecast errors is considered to describe the fuzziness of renewables instead of modeling the output. The modeling of renewables output will make three constraints containing fuzzy variables, which are the supply-demand balance constraint, the spinning reserve constraint, and the static security constraint. If the predicted value is regarded as a deterministic value and the prediction error is utilized to describe the fuzziness of renewables, only the spinning reserve constraint should be considered.

The percentage of renewables prediction error is defined as

$$
\varepsilon \%=\frac{P_{R E S}-P_{R E S}^{F}}{P_{R E S}^{F}} \times 100 \%
$$

where $P_{R E S}$ and $P_{R E S}^{F}$ are the real value and the forecasted value of the output respectively.
The membership function of the prediction error is:

$$
\mu=\left\{\begin{array}{l}
\frac{1}{1+\sigma\left(\frac{\varepsilon}{E_{+}}\right)^{2}}, \quad \varepsilon>0 \\
\frac{1}{1+\sigma\left(\frac{\varepsilon}{E_{-}}\right)^{2}}, \quad \varepsilon \leq 0
\end{array}\right.
$$

where $E_{+}$and $E_{-}$are the average values of positive and negative prediction errors obtained from statistics, respectively; $\sigma$ is the weighting factor.

According to credibility theory, the credibility measure of the fuzzy parameter $\xi$ can be expressed as:

$$
\operatorname{Cr}(\xi \leq \varepsilon)= \begin{cases}1-\frac{1}{2\left[1+\sigma\left(\frac{\varepsilon}{E_{+}}\right)^{2}\right]}, & \varepsilon>0 \\ \frac{1}{2\left[1+\sigma\left(\frac{\varepsilon}{E_{-}}\right)\right]}, & \varepsilon \leq 0\end{cases}
$$

Thus, according to credibility theory, the spinning reserve constraint of the traditional scheduling model

Table 1 Parameters of conventional generators

\begin{tabular}{lllllll}
\hline Node & $\mathrm{a}$ & $\mathrm{b}$ & $\mathrm{c}$ & $P_{G}^{\max }$ & $P_{G}^{\min }$ & $R U(R D)$ \\
\hline 1 & 0.1 & 13.5 & 176.9 & 220 & 100 & 55 \\
2 & 0.1 & 32.6 & 129.9 & 100 & 10 & 50 \\
6 & 0.1 & 17.6 & 137.4 & 20 & 10 & 20 \\
\hline
\end{tabular}


Table 2 Load data of the VPP in $24 \mathrm{~h}$

\begin{tabular}{llllllll}
\hline Time & Load & Time & Load & Time & Load & Time & Load \\
\hline 1 & 175.2 & 7 & 173.4 & 13 & 242.2 & 19 & 246.0 \\
2 & 165.2 & 8 & 177.6 & 14 & 243.6 & 20 & 237.4 \\
3 & 158.7 & 9 & 186.8 & 15 & 248.9 & 21 & 237.3 \\
4 & 154.7 & 10 & 206.9 & 16 & 255.8 & 22 & 232.7 \\
5 & 155.1 & 11 & 228.6 & 17 & 256.0 & 23 & 195.9 \\
6 & 160.5 & 12 & 236.1 & 18 & 246.7 & 24 & 195.6 \\
\hline
\end{tabular}

should be changed into chance constraints containing fuzzy variables.

$$
\operatorname{Cr}\left\{\sum_{i=1}^{N_{G}} u_{i}(t) P_{G i}^{\max }+\sum_{j=1}^{N_{D R}} u_{j}(t) P_{D R j}^{\max }+P_{R E S}^{F}(t)(1+\xi) \geq P_{L}(t)+R(t)\right\} \geq \alpha
$$

where $\alpha$ is the credibility level, which represents the reliability level of the reserve constraint. In the realistic problem, $\alpha$ should be more than 0.5 .

According to the equivalent theorem of credibility theory, (15) can be transformed into an equivalent form as indicated in (16):

$$
\frac{P_{L}(t)+R(t)-P_{R E S}^{F}(t)-\sum_{i=1}^{N_{G}} u_{i}(t) P_{G i}^{\max }-\sum_{j=1}^{N_{D R}} u_{j}(t) P_{D R j}^{\max }}{P_{R E S}^{F}(t)} \leq K_{\alpha}
$$

$$
K_{\alpha}=\inf \left\{K \mid K=\mu^{-1}(2(1-\alpha))\right\}, \forall \alpha \geq 0.5
$$

The equivalent form of the fuzzy chance constraint can be further deduced to the following forms:

$$
\begin{aligned}
& \sum_{i=1}^{N_{G}} u_{i}(t) P_{G i}^{\max }+\sum_{j=1}^{N_{D R}} u_{j}(t) P_{D R j}^{\max }+\left(1+K_{\alpha}\right) P_{R E S}^{F}(t) \geq P_{L}(t)+R(t) \\
& K_{\alpha}=\left|E_{-} \%\right| \sqrt{\frac{2 \alpha-1}{2 \sigma(1-\alpha)}}
\end{aligned}
$$

It can be proved that $K_{\alpha}$ is a monotonously increasing function. Equation (18) illustrates the links between the confidence level of the fuzzy constraint and spinning reserve allocation when taking the fuzziness of RES predicted error into account. It shows that to improve the confidence level of the spinning reserve constraint, a greater spinning reserve should be allocated. The form of the formula is similar to the traditional spinning reserve constraint. Thus, the model can be easily solved by correcting the reserve levels using the regular optimization algorithm with the coefficient $K_{\alpha}$.

\section{Methods}

As introduced above, once chance constraint is transformed into its crisp equivalent, traditional optimization methods can be employed to solve the scheduling problem. Based on the natural selection and genetic manipulation, genetic algorithms (GAs) are heuristic random searching methods, and they possess excellent robustness and commonality. However, there are some shortfalls in the original GA, e.g. the slow convergence speed, falling easily into the locally optimal solution, and so on. A self-adaptive GA method was introduced to improve the performance. The probabilities of crossover and mutation for each generation are adaptively determined, which can overcome the premature convergence and the slow convergence speed in later evolutionary processes.

The crossover and mutation operators are two important genetic operators. In this paper, the crossover

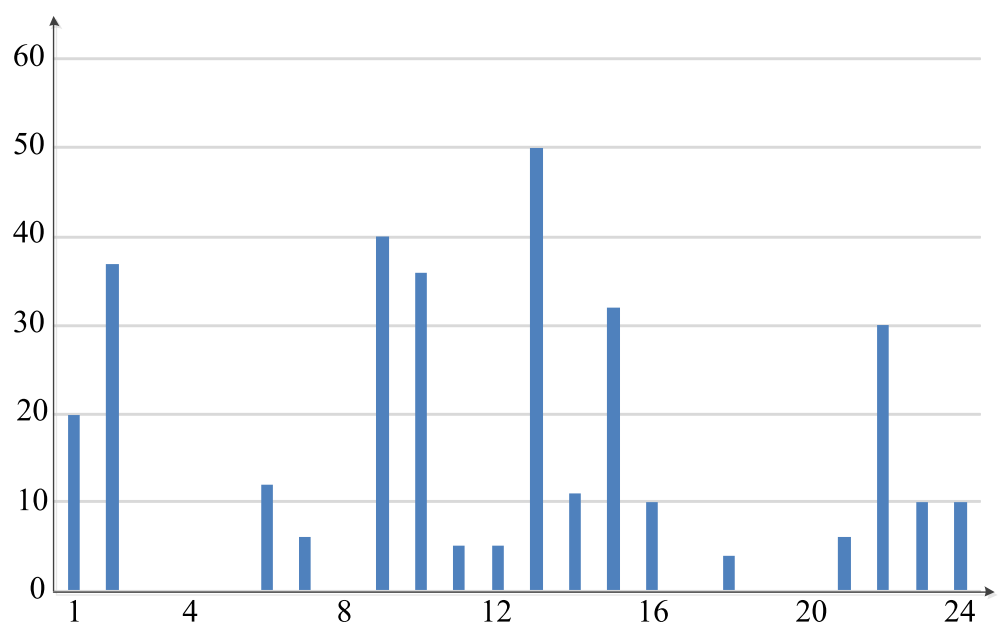

Fig. 2 Forecasted Output of the Wind Farm in $24 \mathrm{~h}$ 
operator adopts double-cut-point crossover and the mutation operator is implemented through power disturbance. The crossover and mutation probabilities adopt self-adaptation mechanism and they will adjust adaptively according to the values of the fitness function [25], as shown in (20) and (21):

$$
\begin{gathered}
P_{c}=\left\{\begin{array}{l}
P_{c 2}-\frac{\left(P_{c 2}-P_{c 3}\right)\left(f^{\prime}-f_{\text {avg }}\right)}{f_{\max }-f_{\text {avg }}}, f^{\prime} \geq f_{\text {avg }} \\
P_{c 1}-\frac{\left(P_{c 1}-P_{c 2}\right)\left(f^{\prime}-f_{\text {min }}\right)}{f_{\text {avg }}-f_{\text {min }}}, f^{\prime}<f_{\text {avg }}
\end{array}\right. \\
P_{m}=\left\{\begin{array}{l}
P_{m 2}-\frac{\left(P_{m 2}-P_{m 3}\right)\left(f^{\prime}-f_{\text {avg }}\right)}{f_{\text {max }}-f_{\text {avg }}}, f^{\prime} \geq f_{\text {avg }} \\
P_{m 1}-\frac{\left(P_{m 1}-P_{m 2}\right)\left(f^{\prime}-f_{\text {min }}\right)}{f_{\text {avg }}-f_{\text {min }}}, f^{\prime}<f_{\text {avg }}
\end{array}\right.
\end{gathered}
$$

where $P_{c}$ and $P_{m}$ are the crossover and mutation probabilities respectively; $f_{\text {max }}, f_{\text {min }}, f_{\text {avg }}$ are the maximum, minimum, and average fitness; $f^{\prime}$ is the greater fitness of the two individuals for genetic operation. Therefore, the superior individual with greater fitness is more likely to be reserved and the inferior individual tends to be transformed into a new one. The improved method could guarantee the population diversity and accelerate its convergence; furthermore, it could avoid the phenomena of premature and slow convergent speed in the later stage of evolution.

\section{Case study}

To evaluate the proposed approach, two test systems are studied. At first, a small VPP consisting of three conventional generators, one $50 \mathrm{MW}$ wind farm and loads (conventional and interruptible) is employed. Then a system consisting of 10 generators and one wind farm is implemented to show the effectiveness of the proposed model.

Table 3 Optimal solutions compared between deterministic and fuzzy chance-constrained model

\begin{tabular}{llllll}
\hline Cost/\$ & $a=0.6$ & $a=0.7$ & $a=0.8$ & $a=0.9$ & $\begin{array}{l}\text { Deterministic } \\
\text { model }\end{array}$ \\
\hline $\begin{array}{l}\text { Start-up cost } \\
\text { of units }\end{array}$ & 400 & 400 & 200 & 161 & 387 \\
$\begin{array}{l}\text { Operation cost } \\
\text { Cost of curtailing }\end{array}$ & 2253 & 934 & 1644 & 1620 & 2333 \\
$\begin{array}{l}\text { load } \\
\text { Total cost }\end{array}$ & 146,033 & 146,015 & 146,124 & 146,122 & 147,020 \\
\hline
\end{tabular}

Table 4 Optimal solutions without curtailing loads

\begin{tabular}{llllll}
\hline Cost/\$ & $a=0.6$ & $a=0.7$ & $a=0.8$ & $a=0.9$ & $\begin{array}{l}\text { Deterministic } \\
\text { model }\end{array}$ \\
\hline $\begin{array}{l}\text { Start-up cost } \\
\text { of units }\end{array}$ & 400 & 400 & 200 & 200 & 200 \\
$\begin{array}{l}\text { Operation } \\
\text { cost }\end{array}$ & 145,854 & 145,865 & 146,080 & 146,096 & 146,287 \\
Total cost & 146,254 & 146,265 & 146,280 & 146,296 & 146,487 \\
\hline
\end{tabular}

\section{Test systems}

The structure of the proposed VPP is shown in Fig. 1. The parameters of the generators and loads in $24 \mathrm{~h}$ are indicated in Tables 1 and 2. The wind farm is integrated into the system at Node 4 , and its forecasted output in $24 \mathrm{~h}$ is shown in Fig. 2. The load at Node 1 is the interruptible load which can be curtailed if necessary. The curtailed load can be regarded as virtual power which can be adjusted continuously and provide the spinning reserve. Its virtual generation capacity ranges from $10 \mathrm{MW}$ to $40 \mathrm{MW}$. The cost paid for the curtailed load is $45 \$ / M W$.

The parameters of the self-adaptive GA are set as follows: the population size $N p=30$; maximum iterations $N_{i t e}=300 ; P_{c 1}=0.85, P_{c 2}=0.5, P_{c 3}=0.2, P_{m 1}=$ $0.09, P_{m 2}=0.05, P_{m 3}=0.01$. Considering the stochastic nature of GA, 20 test trials were conducted for each case.

\section{Results and discussion}

The scheduling model, consisting of (4)-(7), (9)-(11), and (18)-(19), is solved by the self-adaptive GA method in Methods. Apart from the data described in Test systems, different confidence levels are also applied, ranging from 0.6 to 0.9 , in steps of 0.1 . The corresponding costs for satisfying the fuzzy chance spinning reserve constraint are listed in Table 3, including start-up cost, operation cost, cost of curtailing load, and total cost. In addition, the solutions of the fuzzy chance model are compared with those of the traditional deterministic model.

From Table 3, the total costs vary under different confidence levels; especially when $\alpha=0.6, \alpha=0.7, \alpha=0.8, \alpha=$ 0.9 , the total cost are $146,033 \$, 146,015 \$, 146,124 \$$, 146,122 \$, respectively, which are all less than the cost in deterministic model. It can be inferred that the proper selection of confidence levels will help achieve a tradeoff between economy and reliability. However, as the credibility level is increasing gradually from 0.6 to 0.9 , the optimized results (i.e., the total costs) in Table 3 do not show

Table 5 Wind power and the load of the VPP in $6 \mathrm{~h}$

\begin{tabular}{lllllll}
\hline Hour & 1 & 2 & 3 & 4 & 5 & 6 \\
\hline Wind Power/MW & 42 & 63 & 70 & 60 & 58 & 40 \\
Load/MW & 1036 & 1110 & 1258 & 1406 & 1480 & 1628 \\
\hline
\end{tabular}


Table 6 Parameters of conventional generators

\begin{tabular}{lllllll}
\hline unit & $\mathrm{a}$ & $\mathrm{b}$ & $\mathrm{c}$ & $P_{G}^{\max }$ & $P_{G}^{\min }$ & $R U(R D)$ \\
\hline 1 & 0.00043 & 21.60 & 958.20 & 470 & 150 & 80 \\
2 & 0.00063 & 21.05 & 1313.6 & 460 & 135 & 80 \\
3 & 0.00039 & 20.81 & 604.7 & 340 & 73 & 80 \\
4 & 0.00070 & 23.90 & 471.60 & 300 & 60 & 50 \\
5 & 0.00079 & 21.62 & 480.29 & 243 & 73 & 50 \\
6 & 0.00056 & 17.87 & 601.75 & 160 & 57 & 50 \\
7 & 0.00211 & 16.51 & 502.71 & 130 & 20 & 50 \\
8 & 0.00480 & 23.23 & 639.40 & 120 & 47 & 30 \\
9 & 0.10908 & 19.58 & 455.60 & 800 & 20 & 30 \\
10 & 0.00951 & 22.54 & 692.40 & 55 & 55 & 30 \\
\hline
\end{tabular}

a clear monotonic feature. It should be noted that this does not mean that the results of the fuzzy chance model based on credibility theory are confusing. It is because the results are interfered by the demand-side resources.

If the loads are not allowed to be curtailed, the effect of the interruptible load on the solutions will be excluded. Then the total cost of VPP operation in different confidence levels will be 146,254 \$, 146,265 \$, 146,280 \$, 146,296 \& and 146,487 \$ respectively, showing a clear monotonously increasing trend, just as shown in Table 4. This is because, as the credibility level increases, VPP operator needs to spare more reserve capacity to guarantee the growing reliability requirement. Undoubtedly, that would lead to the rise of the total cost. Meanwhile, it is obvious that the total costs in model without curtailing loads are higher than those in original model through comparing the values in Tables 3 and 4. Therefore, the introduction of the demand response (or interruptible loads) helps to improve the economy of the whole system, which is consistent with the current research results.
Furthermore, these results stimulate VPP to incorporate demand response and chance constraint programming into its operation strategy.

To further confirm the effectiveness of the proposed fuzzy chance model, the operation strategy of a larger VPP system in $6 \mathrm{~h}$ is tested. In order to exclude the impact of the interruptible load cost, loads in this system do not participate in the operation. The forecasted output of the wind farm and loads is shown in Table 5, and the parameters of the 10 generators are listed in Table 6 .

The operational costs of VPPs under different confidence levels are depicted in Fig. 3, which are the same as the results in [24], except that confidence levels are set as $1-\alpha$ in this paper. Figure 3 shows the corresponding cost is increasing when the credibility level rises from 0.6 to 0.9 . It is because the confidence level represents the reliability of VPP operation. To reduce the risk resulting from the unpredictable output of renewables, more spinning reserves are required, which will definitely increase the operational cost. The two case studies prove that the proposed model can put forward an optimal operation strategy which can reach the balance between economy and risks/reliability.

\section{Conclusions}

VPP is a promising way to integrate the DERs into the power system. As the output of DERs is usually unpredictable, the scheduling of VPPs has to deal with a number of uncertain variables, which is a fuzzy programming problem. To realize the proper balance between benefits and risks, credibility theory is introduced in the optimal scheduling strategy for VPPs in this paper, thereby proposing a fuzzy chance scheduling model. The concept of the confidence level can quantify the possibility of satisfying the fuzzy chance constraints, which represents the

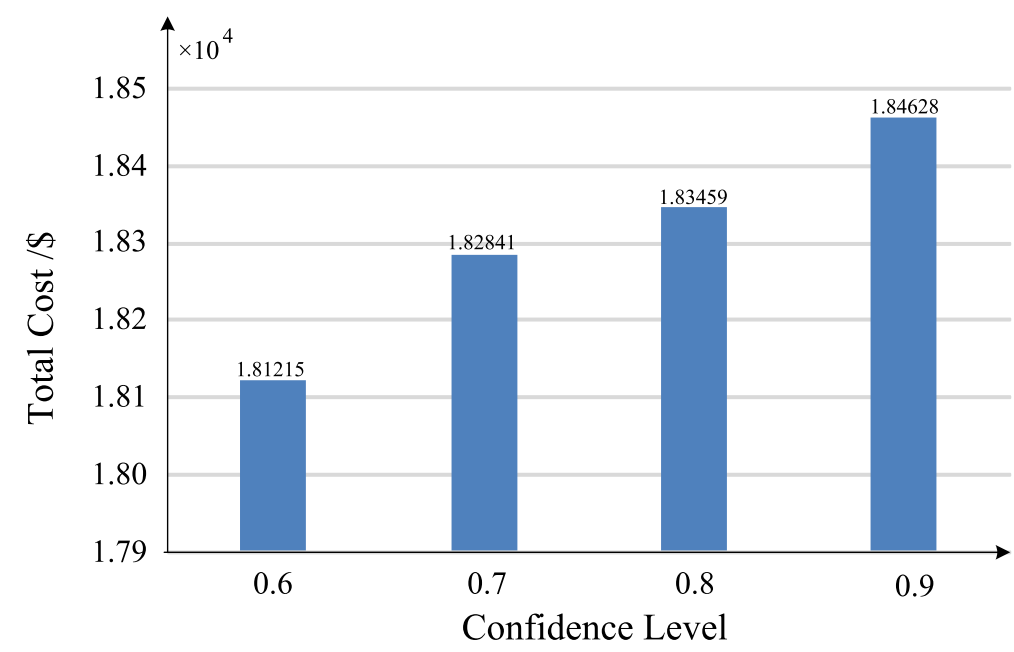

Fig. 3 Total Cost of the VPP under Various Confidence Levels 
risk tolerance of VPPs. Further, through transforming the fuzzy chance constraints into their equivalent forms, the conventional optimization algorithms can be utilized to solve the optimal scheduling problem. The case study proves that the operational cost of VPPs will increase when the confidence level increases. That is to say, unnecessary costs can be reduced when the risk of VPP operation is within tolerance.

\section{Acknowledgment}

This work is supported by the National Natural Science Foundation of China (No. 51577115). Meanwhile, the authors would like to thank the editor and reviewers for their sincere suggestions on improving the quality of this paper.

\section{Authors' contribution}

QA and SF carried out the study of virtual power plants,and drafted the manuscript. All authors read and approved the final manuscript.

\begin{abstract}
About the authors
Q. Ai received the B.S. degree in electrical engineering and automation from Shanghai Jiao Tong University, Shanghai, China, in 1991 and M.S. degree in electrical engineering from Wuhan University, Wuhan, China, in 1994 and Ph.D. degree in electrical engineering from Tsinghua University, Beijing, China, in 1997. After spending one year at Nanyang Technological University, Singapore and two years at University of Bath, UK, he is currently a professor at Shanghai Jiao Tong University, Shanghai, China. His research interests include load modeling, smart grid, and intelligent algorithms. S. L Fan received the B.S. degree in electrical engineering and automation from Sichuan University, Chengdu, China, in 2013. She is currently pursuing the Ph.D. degree in electrical engineering at Shanghai Jiao Tong University, Shanghai, China. Her research interest include virtual power plant operation and optimization.

L. J Piao received the B.S. and M.S. degree in electrical engineering from Shanghai Jiao Tong University, Shanghai, China, in 2012 and 2015, respectively. He is currently pursuing the M.S. degree in electrical engineering at Shanghai Jiao Tong University, Shanghai, China. His current research interests include multi-agent system in smart grids, and electric vehicle coordinated charging using game theory.
\end{abstract}

\section{Competing interests}

The authors declare that they have no competing interests

Received: 12 May 2016 Accepted: 16 May 2016

Published online: 25 June 2016

\section{References}

1. Bamberger $Y$, Baptista J, Belmans R. et al. (2006). Vision and Strategy for Europe's Electricity Networks of the Future: European Technology Platform Smart Grids[R]. Office for Official Publications of the European Communities.

2. Coll-Mayor D, Paget M \& Lightner E. (2007). Future intelligent power grids: analysis of the vision in the European Union and the United States[J]. Energy Policy, 35(4), 2453-2465.

3. Wang C, \& Li P. (2010). Development and challenges of distributed generation, the micro-grid and smart distribution system [J]. Automation of Electric Power Systems, 2, 004.

4. McDermott TE, Dugan RC. (2002). Distributed generation impact on reliability and power quality indices[C]//Rural Electric Power Conference, 2002. IEEE: D3-D3_7.

5. Newman DE, Carreras BA, Kirchner M. et al. (2011). The impact of distributed generation on power transmission grid dynamics[C]//System Sciences (HICSS), 2011 44th Hawaii International Conference on. IEEE: 1-8.

6. Gomez JC, Vaschetti J, Coyos C. et al. (2013). Distributed generation: impact on protections and power quality[J]. Latin America Transactions, IEEE (Revista IEEE America Latina), 11(1), 460-465

7. Fahim SR, Helmy W. (2012). Optimal study of distributed generation impact on electrical distribution networks using GA and generalized reduced gradient[C]//Engineering and Technology (ICET), 2012 International Conference on. IEEE: 1-6.
8. Braun M, \& Strauss P. (2008). A review on aggregation approaches of controllable distributed energy units in electrical power systems[J]. International Journal of Distributed Energy Resources, 4(4), 297-319.

9. Mohammadi J, Rahimi-Kian A, Ghazizadeh MS. (2011). Joint operation of wind power and flexible load as virtual power plant[C]//Environment and Electrical Engineering (EEEIC), 2011 10th International Conference on. IEEE: 1-4.

10. Pudjianto D, Ramsay C, \& Strbac G. (2007). Virtual power plant and system integration of distributed energy resources[J]. Renewable Power Generation, IET, 1(1), 10-16.

11. Pudjianto D, Ramsay C, Strbac G. et al. (2008). The virtual power plant: Enabling integration of distributed generation and demand[J]. FENIX Bulletin, 2, 10-16.

12. Saboori H, Mohammadi M, Taghe R. (2011). Virtual power plant (VPP), definition, concept, components and types[C]//Power and Energy Engineering Conference (APPEEC), 2011 Asia-Pacific. IEEE: 1-4.

13. El Bakari K, Kling WL. (2011). Development and operation of virtual power plant system[C]//Innovative Smart Grid Technologies (ISGT Europe), 2011 2nd IEEE PES International Conference and Exhibition on. IEEE: 1-5.

14. Zdrilić M, Pandžić H, Kuzle I. (2011). The mixed-integer linear optimization model of virtual power plant operation[C]//Energy Market (EEM), 2011 8th International Conference on the European. IEEE: 467-471.

15. Salmani MA, Tafreshi SMM, Salmani H. (2009). Operation optimization for a virtual power plant[C]//Sustainable Alternative Energy (SAE), 2009 IEEE PES/ IAS Conference on. IEEE: 1-6.

16. Ruiz N, Cobelo I, \& Oyarzabal J. (2009). A direct load control model for virtual power plant management[J]. Power Systems, IEEE Transactions on, 24(2), 959-966.

17. Mashhour E, Moghaddas-Tafreshi SM. (2009). Trading models for aggregating distributed energy resources into virtual power plant[C]// Adaptive Science \& Technology, 2009. ICAST 2009. 2nd International Conference on. IEEE: 418-421.

18. Mashhour E, \& Moghaddas-Tafreshi SM. (2011). Bidding strategy of virtual power plant for participating in energy and spinning reserve markets-Part I: Problem formulation[J]. Power Systems, IEEE Transactions on, 26(2), 949-956.

19. Soltani M, Raoofat M, Rostami MA. (2012). Optimal reliable strategy of virtual power plant in energy and frequency control markets[C]//Electrical Power Distribution Networks (EPDC), 2012 Proceedings of 17th Conference on. IEEE: 1-6.

20. Taheri H, Ghasemi H, Rahimi-Kian A. et al. (2011). Modeling and optimized scheduling of virtual power plant[C]//Electrical Engineering (ICEE), 2011 19th Iranian Conference on. IEEE: 1-6.

21. Xue Z, Li G, Zhou M. (2011). Credibility theory applied for estimating operating reserve considering wind power uncertainty[C]//PowerTech, 2011 IEEE Trondheim. Trondheim, Norway: IEEE, 1-8.

22. Zadeh LA. (1965). Fuzzy sets[J]. Information and Control, 8(3), 338-353.

23. Liu B. (2006). A survey of credibility theory[J]. Fuzzy Optimization and Decision Making, 5(4), 387-408.

24. Xin A, \& Xiao L. (2011). Dynamic economic dispatch for wind farms integrated power system based on credibility theory[J]. Proceedings of the CSEE, 31(S1), 12-18.

25. Kuang H, Jin J, \& Su Y. (2006). Improving crossover and mutation for adaptive genetic algorithm[J]. Computer Engineering and Applications, 12, 93-99.

\section{Submit your manuscript to a SpringerOpen ${ }^{\circ}$ journal and benefit from:}

- Convenient online submission

- Rigorous peer review

- Immediate publication on acceptance

- Open access: articles freely available online

- High visibility within the field

- Retaining the copyright to your article

Submit your next manuscript at $>$ springeropen.com 